\title{
Article \\ A Molecularly Imprinted Polymer for Selective Extraction of Phenolic Acids from Human Urine
}

\author{
Marco Mora-Granados ${ }^{1}\left(\mathbb{D}\right.$, David González-Gómez $\left.{ }^{2} \mathbb{(}\right)$ Jin Su Jeong ${ }^{2, *} \mathbb{C}$ and Alejandrina Gallego-Picó ${ }^{1}$ \\ 1 Department of Analytical Sciences, Faculty of Sciences, National University of Distance Education (UNED), \\ 28040 Madrid, Spain; marcomoragra@gmail.com (M.M.-G.); agallego@ccia.uned.es (A.G.-P.) \\ 2 Department of Didactic of Experimental Sciences and Mathematics Education, University of \\ Extremadura (UNEX), 10071 Cáceres, Spain; dggomez@unex.es \\ * Correspondence: jin@unex.es
}

check for updates

Citation: Mora-Granados, M.; González-Gómez, D.; Jeong, J.S.; Gallego-Picó, A. A Molecularly Imprinted Polymer for Selective Extraction of Phenolic Acids from Human Urine. Appl. Sci. 2021, 11, 1577. https://doi.org/10.3390/ app11041577

Academic Editor: Alberto Milani

Received: 11 January 2021

Accepted: 5 February 2021

Published: 9 February 2021

Publisher's Note: MDPI stays neutral with regard to jurisdictional claims in published maps and institutional affiliations.

Copyright: (C) 2021 by the authors. Licensee MDPI, Basel, Switzerland. This article is an open access article distributed under the terms and conditions of the Creative Commons Attribution (CC BY) license (https:/ / creativecommons.org/licenses/by/ $4.0 /)$.
Featured Application: In this research, a procedure to prepare a molecular imprinted polymer is presented for the selective extraction of phenolic acids from human urine samples.

Abstract: Studies for monitoring the bioavailability of dietary flavonoid compounds generate great interest. Among them, low-molecular-weight phenolic acids, secondary metabolites present in colonic catabolism and urinary excretion, have been proposed as biomarkers of polyphenol intake. Using 4-hydroxyphenylacetic acid as a template, a molecularly imprinted polymer (MIP) was synthesized for selective extraction of these hydroxylated metabolites from human urine samples and posterior analysis in an HPLC-DAD-MS system. Polymers were characterized by Scanning electron microscopy (SEM), Attenuated total reflection-Fourier transform infrared spectroscopy (ATR-FTIR), BrunauerEmmett-Teller (BET) method, and binding experiments. MIP presents specific recognition ability for template and analogues molecules. This capacity of recognition and the $\mathrm{pH}$ dependence of the binding strength was also studied. The method was validated over a concentration range of $0.25-$ $40 \mathrm{mg} / \mathrm{L}, \mathrm{r}^{2}>0.995$. In the optimized conditions, the recovery value was $94 \%$ with $\mathrm{RSD} 1.2 \%$. The Limit of Detection (LOD) and Limit of Quantification (LOQ) were 1.22 and $3.69 \mathrm{mg} / \mathrm{L}$, respectively. In our knowledge, it is the first time that this methodology is applied to analyze urinary catabolites of the polyphenol compound and to provide a specific method and simple analysis alternative. The selective extraction of these metabolites improves the application and results obtained by other less sensitive analysis methods than the validation method. It also facilitates the development of new screening methods.

Keywords: molecularly imprinted polymer; polyphenol metabolites; solid phase extraction; human urine; phenolic acids; HPLC-DAD-MS

\section{Introduction}

Diets rich in fruits and vegetables are related to positive effects on health and a lower risk of diseases (cardiovascular disorders, cancer, or neurodegeneration) [1]. Flavan-3-ols are the most common group of flavonoids in the human diet and the analysis and quantification of these metabolites could report valuable information to assess their absorption mechanisms and bioavailability. Flavan-3-ols can be found in fruit, vegetables, legumes, beverages (cider, coffee, beer, tea, and wine), or chocolate, and also are added as functional ingredients in processed foods or dietary supplements. In the colon, dietary phenolic compounds can be converted into smaller phenolic acid by microorganisms and may contribute to health benefits. These benefit effects, previously related to the simple direct antioxidant hypothesis of an understanding of the complex effects on molecular targets and enzymes in specific cell types, now have undergone and the importance of the microbiome is inherently integrated with flavonoid metabolism and bioactivity [1]. In the literature, several metabolites are proposed as biomarkers for monitoring the bioavailability and human dietary 
intake of polyphenol compounds after consumption, and low-molecular-weight phenolic acids, secondary metabolites present in colonic catabolism and urinary excretion, have been mostly suggested for this purpose in urine and feces [2-5]. Consequently, the grand interest in knowing the bioavailability of dietary flavonoids and phenolic compounds has stimulated analytical methods development. In the last years, many methods have been published for determination of polyphenol and its hydroxylated metabolites in plants, animal, and human samples [6], but currently chromatographic techniques coupled to mass spectrometry are being widely used [2,7]. Furthermore, other new strategies based on chemometric tools have been developed [8]. Nevertheless, the most important challenge in the analysis of polyphenols and its metabolites is the extraction and isolation of compounds from the complex matrices. Recent trends in sample preparation and purification include the development of advanced adsorbent materials and accordingly, several analytical methodologies have been proposed to improve the sensitivity and specificity in the polyphenol compound determination [9-13]. Molecular imprinted polymers (MIP) have attracted wide attention and attained significant applications in selective extraction of polyphenols compounds and hydroxylated metabolites in plants, natural products, foodstuffs, or biological and environmental samples. However, MIP applications in the analytical determination of polyphenols metabolites have been limited and few of us used phenolic acid as a template [10,13-20]. PCA has been used as template for the synthesis of an MIP for the extraction analogues compounds from plant in an Molecularly Imprinted Solid Phase Extraction (MISPE) procedure $[13,14]$ or used as High-Performance Liquid Chromatography (HPLC)stationary phase to analyze synthetic samples of red wine $[15,16]$. 4-HBA-MIP has also been used in the stationary phase $[16,19]$ and imprinting studies were carried out [17]. The 4-HBA MISPE procedure has also been proposed for the selective extraction of polyphenols from olive mill waste waters [18]. In our knowledge, only one work has reported a MIP synthesis using 4-HPA as a template and HPLC columns application and mechanism of recognition was discussed theoretically [19,21]. On the other hand, only one study has applied MIP for the analysis in urine. 4-methylhippuric acid has been used as a template for the synthesis of an MIP for the extraction of hippuric and 4-methylhippuric acids for the assessment exposure of toluene and xylenes [22]. Main functional monomers used in these molecular imprinting procedures were $4-\mathrm{Vpy}$, acrylamide, and methacrylic acid [10,13-22]. To our knowledge, only one work reported the application of MIP for the phenolic acid analysis in urine, but with a different purpose, the assessment exposure of toluene and xylenes [22]. In our study, 4-hydroxyphelnylacetic acid (4-HPA), excreted catabolite of polyphenols compounds, was chosen as a template of molecularly imprinted polymers. Synthesized MIP was used as sorbent in a SPE procedure (MISPE) for extraction and isolation of 4-HPA and analogues. Once the MISPE parameters were optimized, the procedure was validated using spiked human urine samples.

\section{Materials and Methods}

\subsection{Chemicals and Solutions}

Standards of phenolic acid, 3-hydroxyphenylacetic acid (3-HPA), 4-hydroxyphenylacetic acid (4-HPA), protocatechuic acid (PCA), 4-hydroxybenzoic acid (4-HBA), syringic acid (SyA), and hippuric acid (HA) were purchased from Sigma-Aldrich (Madrid, Spain), Acros Organics (Geel, Belgium), and HWI Analytik (Rülzheim, Germany). Other reagents used for polymerization as ethylene glycol dimethacrylate (EGDMA), 2,2'-azobis(2-methylpropionitrile) (AIBN) were obtained from Sigma-Aldrich (Madrid, Spain) and 4-vinylpyridin (4-Vpy) were purchased from Merck-Schuchardt (Hohenbrunn, Germany). HPLC-grade solvents used were acetonitrile $(\mathrm{ACN})$ and methanol $(\mathrm{MeOH})$, they were purchased from Scharlau (Barcelona, Spain). Ultrapure water $(18.2 \mathrm{M} \Omega / \mathrm{cm}$ quality) was obtained using a Milli-Q water system (Millipore Ibérica, Spain) and used for all dilutions. All other reagents used were of analytical grade or better.

Stock solution of 6 metabolites $(1000 \mathrm{mg} / \mathrm{L})$ was prepared in water and stored at $4{ }^{\circ} \mathrm{C}$ till their usage. Ultrasonic bath was used in preparation solutions to aid analyte dissolution. 
All working solutions of analytes were daily prepared by dilution in water of stock solutions. All solutions were stored in amber-colored flasks to prevent photodegradation.

\subsection{Samples}

Phenolic free urine samples were collected from healthy breastfed newborns. After centrifugation and filtration using Whatman PPW/GMF polypropylene filters $(0.45 \mu \mathrm{m})$, the samples were stored without preservatives at $-20^{\circ} \mathrm{C}$ until use. Before processing, samples were allowed to thaw at room temperature and then homogenized by gently shaking. For the analysis, urine was appropriated spiked with the working solutions to cover the calibration range: 0.25 to $40 \mathrm{mg} / \mathrm{L}$. Spiked urine samples were stored for $30 \mathrm{~min}$ to foster analyte-urine interaction. In these conditions, $\mathrm{pH}$ of samples was around 6.8 . Previous $\mathrm{pH}$ studies were carried out for each analyte, demonstrating that the MISPE procedure improved when the $\mathrm{pH}$ of samples was 3 , and in addition, good recovery was obtained. More acidic or basic media produced a reduction of the recovery and consequently, the $\mathrm{pH}$ of the urine was adjusted to 3 , using hydrochloric acid.

\subsection{Chromatographic Conditions}

Chromatographic analysis was performed on an Agilent LC 1200 series equipped with a thermostatted column compartment and a standard autosampler coupled to a diode array detector on-line with a mass detector (Agilent 6100 series). UV spectra were used to confirm the identity of phenolic acid.

Separation was achieved on a Zorbax Eclipse XDB-C18 $(150 \times 4.6 \mathrm{~mm}, 5 \mu \mathrm{m})$ linked to a security guard cartridge Zorbax Eclipse XDB-C18 $(12.5 \times 4.6 \mathrm{~mm}, 5 \mu \mathrm{m})$ from Agilent Technologies (Spain). The chromatographic separation was carried out at constant temperature $\left(25^{\circ} \mathrm{C}\right)$. Mobile phase was constituted with acetonitrile and water acidified with $0.1 \%$ formic acid (10:90) in isocratic mode. The flow rate was $0.4 \mathrm{~mL} / \mathrm{min}$ and the injection volume, $20 \mu \mathrm{L}$. MS analyses were performed in the negative mode and the ESI parameters were as follows: capillary voltage, $-3 \mathrm{kV}$; nitrogen nebulizer ( $35 \mathrm{psi}$ ) and drying gas flow $(12.0 \mathrm{~L} / \mathrm{min})$ at $350^{\circ} \mathrm{C}$. First, a solution with all of standards was infused in scan mode, so the deprotonated molecule and the most intense product ion of each compound were recorded and selected for optimization and quantitative analysis of samples (SIM mode). The optimized cone voltage for all analytes was $40 \mathrm{~V}$. The chromatographic procedure was optimized to analyze the six analytes. The DAD was operated in $280 \mathrm{~nm}$ wavelength. Mass spectrometric data were collected between 10 and $28 \mathrm{~min}$. Chromatographic data were recorded and integrated using Chemstation (rev. B.04.02) for LC Agilent system.

\subsection{Synthesis of Molecularly Imprinted Polymers}

MIP was synthesized using $152 \mathrm{mg}$ 4-HPA as template, 4-Vpy $(431 \mu \mathrm{L})$ as functional monomer, EGDMA $(3.77 \mathrm{~mL})$ as crosslinker, AIBN $(50 \mathrm{mg})$ as polymerization reaction initiator, and $\mathrm{ACN}(10 \mathrm{~mL})$ as porogen. Porogen volume influences polymer morpholgy, and according to some authors, dilution conditions lead the size of the gel particles in a crosslinking copolymerization [23]. The porogen volume has been determined by preliminary study (70.4 vol \% relative to the total volume). The polymerization was carried out in a $20 \mathrm{~mL}$ glass vial, and a molar ratio of 1:4:20 (template/4-Vpy/EGDMA) was maintained in excess of AIBN. The pre-polymerization mixture was purged with nitrogen during $15 \mathrm{~min}$ and then was sonicated during $15 \mathrm{~min}$ in an ultrasonic bath (Selecta Ultrasons, Barcelona, Spain). The solution was cooled on an ice bath and purged with nitrogen for $15 \mathrm{~min}$, in order to remove oxygen and establish an inert atmosphere. Afterwards, the glass vial was sealed and placed in a thermostatted bath. The polymerization process was thermally initiated and maintained at $60^{\circ} \mathrm{C}$ for a period of $24 \mathrm{~h}$. The monolithic polymer obtained was crushed and sieved to give particles of size between 100 and $50 \mu \mathrm{m}$, suitable for SPE applications. An ETHOS SEL microwave oven (Milestone, Sorisole, Italy) was used to remove the template from the imprinted polymer. Standard microwave-assisted extraction (MAE) conditions were as follows: MIP and $30 \mathrm{~mL}$ of washing solution (methanol 
containing $10 \% v / v$ acetic acid) were placed in the extraction vessels and exposed to MAE over a 5 min heating ramp period up to $100{ }^{\circ} \mathrm{C}$. This temperature was then maintained for $20 \mathrm{~min}$. After extraction, the vessel content was transferred to a vacuum filtration system for removal of the solvent. This process was performed three times in order to ensure the total extraction of the template and washing solutions were kept for posterior extraction efficiency studies. Finally, the polymer particles were dried at $60{ }^{\circ} \mathrm{C}$ in a hot air oven and stored at room temperature prior to use. Non-imprinted polymers (NIP) were prepared in the same manner as the MIP polymer but in the absence of template (4-HPA).

\subsection{MIP and NIP Characterizations}

In this work, the morphology of polymers was examined. Porosity measurements surface areas of MIP and NIP were measured by nitrogen sorption porosimetry using Micrometrics ASAP 2020 equipment (Micrometrics Instruments, Norcross, GA, USA). The specific surface areas were calculated using the Brunauer-Emmett-Teller (BET) method, and pore volume data were calculated according to the Barret-Joyner-Halenda (BJH) method. Before the BET measurement, the particles were heated at $120^{\circ} \mathrm{C}$ for $2 \mathrm{~h}$ in vacuum for remove gases and vapors that may have become physically adsorbed onto the particle surface. Surface characterization of polymer particles is of high importance to investigate the surface area and the size/volume characteristics of the pores since these parameters affect the binding of analytes. Scanning electron microscopy (SEM) is performed for MIP and NIP particles visualization (SEM, S-3000N, Hitachi, Japan). All samples were sputtercoated with Au/Pd before SEM analysis (Polaron Range SC7620). Chemical structure of polymers was characterized by Fourier transform infrared spectroscopy (FTIR) from 7000 to $400 \mathrm{~cm}^{-1}$, operating in the ATR mode, with 64 scans resolution at $4 \mathrm{~cm}^{-1}$, using an FT/IR-4100A spectrophotometer with the accessory ATR PRO ONE (JASCO, Spain). The sample was directly added on the device without any treatments.

\subsection{Batch Mode Binding Experiments}

An adsorption experiment was carried out to study binding properties of MIP and NIP. In this batch experiment, $20 \mathrm{mg}$ of MIP or NIP were added to $2.5 \mathrm{~mL}$ of standard solution of 4-HPA and analogues with various concentrations ranging from 0.5 to $1000 \mathrm{mg} / \mathrm{L}$. All solutions of analytes were prepared by dilution in water of stock solutions. The mixture was incubated for $24 \mathrm{~h}$ at room temperature stirring at $450 \mathrm{rpm}$. The supernatants and polymer were isolated by centrifugation and filtration $(0.45 \mu \mathrm{m})$. The concentration of unbound analytes in the supernatant was analyzed by HPLC-DAD-MS. All the model parameters were evaluated by both non-linear regression and linear least-squares method using OriginPro v. 8.0 software of OiginLab Corp. (Northampton, UK).

\subsection{MISPE Cartridge Preparation and Extraction Conditions}

MISPE cartridge was prepared packing the resulting microparticles polymer (100 mg) into an empty SPE polypropylene cartridge of $3 \mathrm{~mL}$ between two frits with $20 \mu \mathrm{m}$ pore size (Agilent Technologies). A similar procedure was used to prepare the NIP-SPE cartridge. Before conditioning, cartridges were washed and dried. A vacuum SPE manifold system (Varian Technologies, Palo Alto USA) with vacuum control press-pump (Selecta, Spain) was used in the SPE procedure.

For optimum conditions, the MISPE cartridge was conditioning with $2.0 \mathrm{~mL}$ of water and then $2.0 \mathrm{~mL}$ of acetronitrile and loading with $0.5 \mathrm{~mL}$ of a 4-HPA standard solution $(4 \mathrm{mg} / \mathrm{L})$ in water. The washing step was carried out using $1.0 \mathrm{~mL}$ of water. The analyte retained in the cartridge was eluted using $1.5 \mathrm{~mL}$ of acetonitrile with $1 \%$ formic acid. Solutions were added to the cartridge and then allowed to pass through the column under vacuum conditions. Both the washing and elution fractions were collected and dried under a gentle nitrogen stream at room temperature. The residues were reconstituted with the adequate volume of mobile phase previous HPLC-DAD-MS analysis. 


\subsection{Analytical Figure of Merit}

The MISPE-LC-DAD-MS method developed was validated in terms of linearity, reproducibility, and detection and quantification limits (LOD and LQO). Linearity was expressed by means of the coefficient of determination $\left(\mathrm{r}^{2}\right)$. The LOD and LOQ values were calculated at signal-to-noise ratios of 3 and 10 respectively, following International Union of Pure and Applied Chemistry (IUPAC) recommendations. Finally, the reproducibility of the method was evaluated with the relative standard deviation parameter RSD in term of reproducibility (inter-day precision and intra-day precision) at three levels of fortification: 2, 20, and $40 \mathrm{mg} / \mathrm{L}$.

\section{Results and Discussion}

\subsection{MIP Design, Template, and Analogues Selection}

MIPs are highly reticulated network polymers consisting of a common matrix structure and binding sites formed by a template present during polymer synthesis and monomers are chosen in order to complement functional groups of a template molecule. After remove of the template, binding sites remain that are capable of rebinding the template with high affinity and selectivity. These sites can interact with the template molecules in a noncovalent way, such as via coulombic interactions, hydrogen bonding, ion coordinating, $\pi-\pi$ stacking, hydrophobic and van der Waals forces, or by reversible formation of covalent bonds [24]. Non-covalent imprinting is efficient for low-molecular-weight compounds and templates containing acids are often well targeted using basic functional monomers such as vinylpyridine (4-Vpy), or amide monomers such as acrylamide (MA) $[18,24]$. Some authors suggested that the best recognition ability depended on the pKa relationship between monomer and template. The basic character of $4-\mathrm{Vpy}(\mathrm{pKa}=5.4)$ makes it a good candidate for phenolic acid templates [10,18,22]. Sun et al. also related the rigid structure of templates to the recognition ability of synthesized MIP [19]. Templates with high molecular rigidity (e.g., 4-HBA or 4-HPA) showed a much stronger imprinting effects. Therefore, MIP was synthesized using 4-HPA as a template, 4 -Vpy as a functional monomer, ethylene glicol dimethacrylate (EGDMA) as a crosslinker, 2,2'-azobis(2-methyl-propionitrile (AIBN) as polymerization reaction initiator. The polymerization was carried out in a molar ratio of 1:4:20 (template/4-Vpy/EGDMA). Porogen plays an important role in the polymer morphology and also affects the ionic interactions and hydrogen bonds between target and the functional monomer [23]. An aprotic and polar solvent such as ACN was chosen as porogen. The optimized volume of porogen was $10 \mathrm{~mL}$.

Recently, a large number of phenolic acids and other catabolites have been detected in urine after flavonoid intake [2,5,25-27]. Maximum concentration of phenolic metabolites depends of the parent flavonoid and the excretion time [25]. We chose 4-HPA as the target and template analyte because it is determined in higher concentration in urine and presents more flexibility that other phenolic acid molecules. Additionally, maximum peak excretions by 4 -HPA were observed between $4-5 \mathrm{~h}$ post-consumption [25]. On the other hand, the sorption properties of molecularly imprinted polymers with imprints of phenolic acids and their derivatives were found to depend on the number and location of hydroxyl and carboxyl groups [17]. In order to assess the MIP, we chose other well-known metabolites as analogues present in urine (Figure 1). We chose an isomer of a template, 3-hydroxyphelacetic acid (3-HPA). Furthermore, we chose three compound with structural similarity: 4-hydroxybenzoic acid (4-HBA), 3,4-dihydroxybenzoic acid (protocatechuic acid, PCA), and 4-hydroxy-3,5-dimethoxybenzoic acid (syringic acid, SyA). Further, 4-HBA also has structural similarity with gallic acid, caffeic acid, and vanillic acid, which are well-known metabolites. The last, hippuric acid (HA), proposed as a biomarker for dietary flavonoid intake [3], was included. 
4-HPA<smiles>O=C(O)Cc1ccc(O)cc1</smiles>

SyA<smiles>COc1cc(C(=O)O)cc(OC)c1O</smiles>

HA<smiles>O=C(O)CNC(=O)c1ccccc1</smiles>

3-HPA<smiles>O=C(O)Cc1cccc(O)c1</smiles>

PCA<smiles>O=C(O)c1ccc(O)c(O)c1</smiles>

4-HBA<smiles>O=C(O)c1ccc(O)cc1</smiles>

Figure 1. Chemical structures of the template (4-HPA) and its analytes analogues (3-HPA, PCA, 4-HBA, SyA, and HA).

\subsection{Polymer Characterizations}

Polymer structures (MIP and NIP) were studied by BET and SEM. In the BET procedure, nitrogen adsorption isotherm determination is used to obtain information about the total pore volume and the surface area [26]. BJH desorption pore volumes obtained were $4.80 \mathrm{~cm}^{3} / \mathrm{g}$ for MIP and $0.75 \mathrm{~cm}^{3} / \mathrm{g}$ for NIP. The specific surface determined by BET relates to the reactive area (total surface area) as all porous structures adsorb the small gas molecules. BET surface areas were found to be 274.8 and $230.7 \mathrm{~m}^{2} / \mathrm{g}$ for MIP and NIP, respectively. MIP and NIP showed a similar physisorption isotherms and similar surface areas, which could indicate that MIP had a similar structure to NIP. However, when considering the pore volume, the MIP exhibited 6 time more pore volume than the corresponding NIP. Figure 2 shows BET data of surface areas and the linear plot obtained for MIP and NIP for N2 at $77 \mathrm{~K}$.

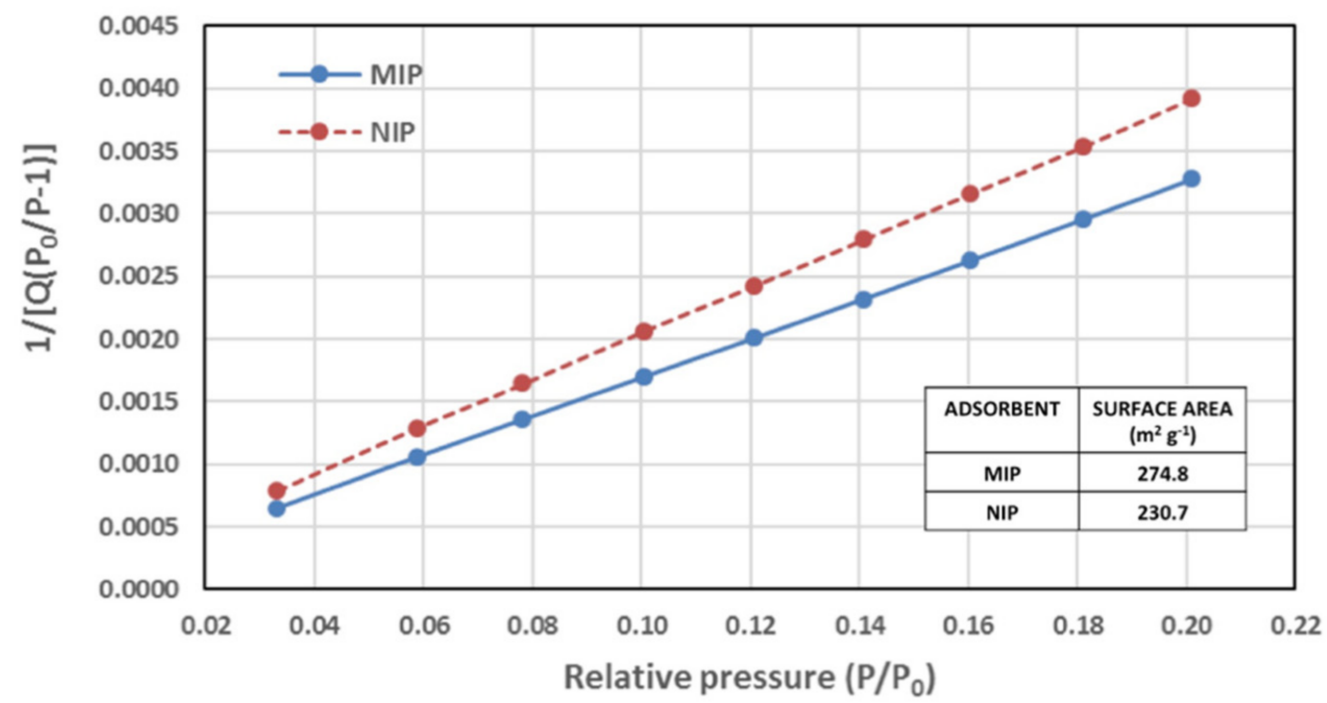

Figure 2. BET data of surface areas and linear plot obtained for molecularly imprinted polymer (MIP) and nonimprinted polymers (NIP) for $\mathrm{N}_{2}$ at $77 \mathrm{~K}$ (Q is quantity adsorbed in $\mathrm{cm}^{3} / \mathrm{g}$ ). 
MIP and NIP particles morphology were explored by SEM. SEM is especially useful for convenient inspections of grain structures. Figure 3 presents the SEM microscopic images of the MIP particles by crushed polymer monolith obtained by bulk polymerization, displaying a non-spherical shape (Figure 3A). The textural surface of polymers revealed a typical pattern on the imprinted (Figure 3B) and non-imprinted surfaces (Figure 3C). A different roughness and smoother surface of the NIP was observed [16,28].

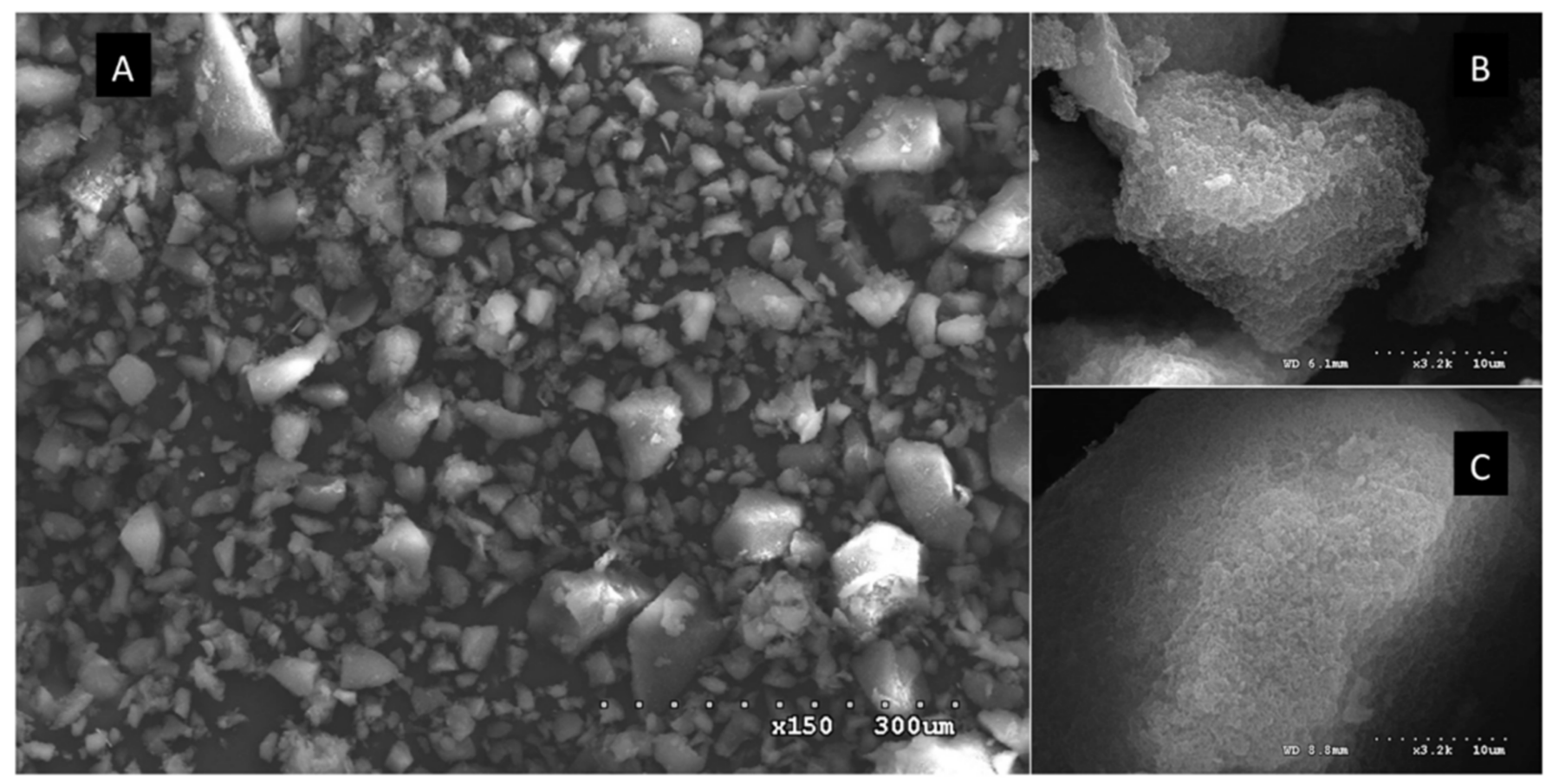

Figure 3. SEM microscopic images of crushed polymers produced by bulk polymerization method. (A) and (B) show MIP particles, magnification $\times 150$ and $\times 3200$, respectively, while $($ C) shows NIP particles, magnification $\times 3200$.

Figure 4 presents ATR-FTIR spectra of the MIP, NIP, and the template (4-HPA). ATRFTIR analysis provides information about the state of the polymerization $[15,16]$. The non-existence of bands in the region $1680-1640 \mathrm{~cm}^{-1}$ indicates the absence of vinyl group in the polymers, and confirms their total polymerization. 4-HPA presents characteristic bands at 3200 and $1690 \mathrm{~cm}^{-1}$ associated with the $\mathrm{O}-\mathrm{H}$ and $\mathrm{C}=\mathrm{O}$ stretching of carboxylic acid; $1400 \mathrm{~cm}^{-1}$ is assigned to vibrations of the benzene ring, and $1270 \mathrm{~cm}^{-1}$ corresponds to $\mathrm{O}-\mathrm{H}$ stretching of phenols. The absence of characteristic bands for template also indicates a successful extraction process $[15,16]$. Some authors are associated other characteristic MIP and NIP bands to crosslinker or the existence of poly(EGDMA) [14,22]. MIP and NIP present similar spectra, except for the intensity of the $\mathrm{C}=\mathrm{O}$ stretching vibration band $\left(1724 \mathrm{~cm}^{-1}\right)$. In NIP, this band is lower than that of MIP [14,22]. 


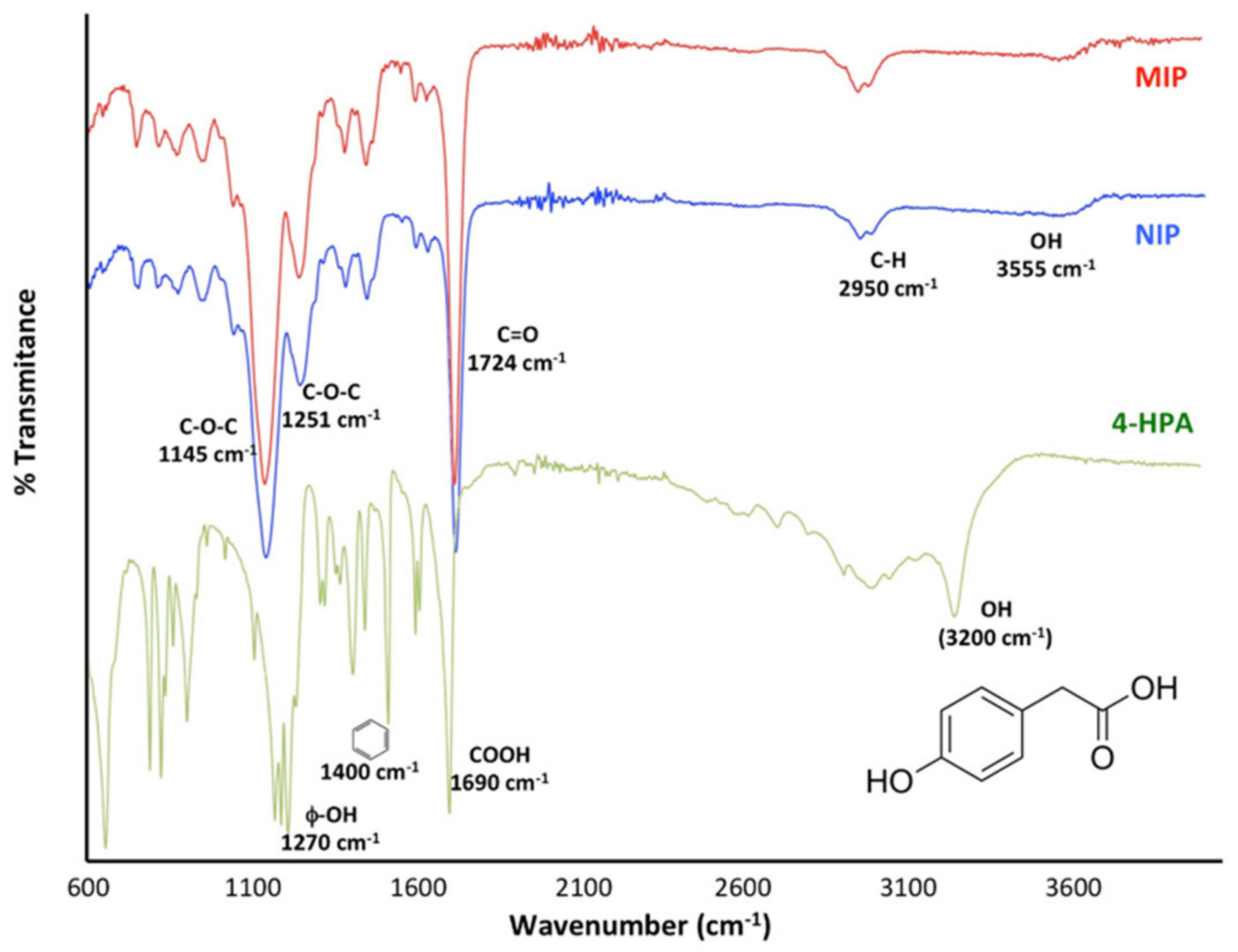

Figure 4. ATR-FTIR spectra for MIP, NIP, and 4-HPA.

\subsection{Binding Properties and Adsorption Isotherms}

For evaluation of the imprinting ability of phenolic acids, its imprinting factors (IF) were evaluated. IF compares the adsorption ability of MIP to NIP and was obtained from the ratio of the adsorption capacity of MIP (QMIP) to NIP ( $\left.\mathrm{Q}_{\mathrm{NIP}}\right)$. Results indicate that the template presents higher adsorption capacity and stronger interaction than its structural analogues because the MIP specific sites are complementary in shape, size, and spatial distribution to template. Consequently, the highest IF is presented by 4-HPA (the template), followed by its isomer (3-HPA), IF values were 3.5 and 3.2. Other analogues show intermediate IF values. SyA IF value was 2.9, PCA and 4-HBA IF values were 2.6 and 2.4, respectively. On the other hand, the lowest IF is presented by HA, IF was 2.2. It can also be seen that IF values are more than 1.0, and MIPs exhibit an imprinting effect for studied compounds with chemical structures similar to that of the 4-HPA imprinting template [17]. In MIP, adsorption capacities were influenced by the presence, number, and position of hydroxyl and carboxyl groups in the aromatic ring $[17,29]$. On the other hand, the position of the hydroxyl group in relation to the carboxyl group also influences selectivity of MIP [16].

Modeling the adsorption isotherm data is an essential way for predicting and comparing the adsorption performance. In this work, Langmuir and Freundlinch isotherm models were used to analyze the adsorption data of MIP [30]. Table 1 resume Langmuir and Freundlich isotherm parameters for MIP and the different phenolic acids. Standard deviation (SD) and the correlation coefficient (R) were used to determine the best-fitting isotherm to the experimental data. Results suggest that Langmuir model was more applicable to estimate the affinity distributions than the Freundlich model according to the correlation coefficients (R). These clearly show that the MIP values of $Q_{\max }$, obtained in the Langmuir model, were higher for 4-HPA and 3-HPA. 
Table 1. Langmuir and Freundlich isotherm parameters obtained by non-linear fitting for the MIP polymer.

\begin{tabular}{|c|c|c|c|c|c|c|c|c|c|c|c|c|c|c|c|}
\hline \multirow{2}{*}{\multicolumn{3}{|c|}{ Model }} & \multirow{2}{*}{ Parameters } & \multicolumn{2}{|c|}{ 4-HPA } & \multicolumn{2}{|c|}{ 3-HPA } & \multicolumn{2}{|c|}{ 4-HBA } & \multicolumn{2}{|c|}{ PCA } & \multicolumn{2}{|c|}{ SyA } & \multicolumn{2}{|c|}{ HA } \\
\hline & & & & Value & S.D. & Value & S.D. & Value & S.D. & Value & S.D. & Value & S.D. & Value & S.D. \\
\hline \multicolumn{3}{|c|}{ Langmuir } & \multirow{2}{*}{$\begin{array}{c}\mathrm{A}=1 / \mathrm{Qmax} \\
\mathrm{B}=1 /\left(\mathrm{K}_{\mathrm{L}} \mathrm{Qmax}\right) \\
\mathrm{R}\end{array}$} & \multirow{2}{*}{$\begin{array}{l}0.0076 \\
10.173 \\
0.998\end{array}$} & \multirow{2}{*}{$\begin{array}{l}0.013 \\
0.179 \\
-\end{array}$} & \multirow{2}{*}{$\begin{array}{c}0.009 \\
11.644 \\
0.999\end{array}$} & \multirow{2}{*}{$\begin{array}{l}0.008 \\
0.101\end{array}$} & \multirow{2}{*}{$\begin{array}{c}0.024 \\
12.186 \\
0.999\end{array}$} & \multirow{2}{*}{$\begin{array}{l}0.010 \\
0.136\end{array}$} & \multirow{2}{*}{$\begin{array}{c}0.025 \\
10.025 \\
0.999\end{array}$} & \multirow{2}{*}{$\begin{array}{l}0.005 \\
0.068\end{array}$} & \multirow{2}{*}{$\begin{array}{l}0.017 \\
9.810 \\
0.999\end{array}$} & \multirow{2}{*}{$\begin{array}{l}0.004 \\
0.053\end{array}$} & \multirow{2}{*}{$\begin{array}{c}0.009 \\
17.492 \\
0.997\end{array}$} & \multirow{2}{*}{$\begin{array}{l}0.026 \\
0.351\end{array}$} \\
\hline$\frac{1}{Q}=$ & $\frac{1}{\mathrm{Q}_{\max } \mathrm{K}_{\mathrm{L}}}$ & $\times \frac{1}{\mathrm{Ce}}+\frac{1}{\mathrm{Q}_{\max }}$ & & & & & & & & & & & & & \\
\hline \multirow{3}{*}{\multicolumn{3}{|c|}{$\begin{array}{c}\text { Freundlich } \\
\log Q=\log K_{F}+\frac{1}{n} \log C_{e}\end{array}$}} & $\mathrm{~A}=\log \mathrm{K}_{\mathrm{F}}$ & -0.875 & 0.094 & -0.914 & 0.058 & -0.908 & 0.045 & -0.809 & 0.065 & -0.822 & 0.056 & -1.096 & 0.081 \\
\hline & & & $\mathrm{b}=1 / \mathrm{n}^{\mathrm{r}}$ & 0.898 & 0.042 & 0.872 & 0.026 & 0.827 & 0.020 & 0.8139 & 0.029 & 0.845 & 0.030 & 0.891 & 0.038 \\
\hline & & & $\mathrm{R}$ & 0.992 & - & 0.993 & - & 0.995 & - & 0.989 & - & 0.991 & - & 0.986 & - \\
\hline
\end{tabular}

$\mathrm{C}_{\mathrm{e}}$ is the concentration of solution at equilibrium $(\mathrm{mg} / \mathrm{L}), \mathrm{Q}$ is the corresponding adsorption capacity $(\mathrm{mg} / \mathrm{g}), \mathrm{Q}_{\mathrm{max}}(\mathrm{mg} / \mathrm{g})$ and $\mathrm{K}_{\mathrm{L}}(\mathrm{L} / \mathrm{mg})$ are constants which are related to adsorption capacity and energy or net enthalpy of adsorption, respectively. $\mathrm{K}_{\mathrm{F}}$ and $\mathrm{n}$ are Freundlich constants which correspond to adsorption capacity and adsorption intensity, respectively.

\subsection{Optimization of MISPE Procedure}

In this study, volume and concentration of sample, loading, washing, and elution solvents and volumes were optimized. Before the sample loading, the cartridge was conditioning with $2 \mathrm{~mL}$ of water and then $2 \mathrm{~mL}$ of ACN. The loading solvent is chosen in order to allow rebinding of the analyte to specific sites, whereas the elution solvent has to be optimized according to its ability to disrupt the analyte-polymer interaction. In accordance with the literature, the solvent used as porogen during polymerization was chosen as the loading solvent. Then, different concentrations of 4-HPA (4 to $100 \mathrm{mg} / \mathrm{L}$ ) in water were prepared to assess loading concentration. In this concentration range, results obtained did not show significant difference in the recovery. Therefore, lower concentration of $4 \mathrm{mg} / \mathrm{L}$ was chosen as the working concentration. Loading was carried out maintaining the sample onto the cartridge during $5 \mathrm{~min}$ and then it was passed through the column under vacuum conditions. Subsequently, different sample volumes $(0.5-2 \mathrm{~mL})$ were tested to evaluate the loading. The retained 4-HPA was evaluated and calculated as the difference between 4-HPA total amounts loaded onto the cartridge initially, and collected 4-HPA in the fractions from the loading and washing steps. In each process, the obtained extracts were injected into the HPLC-DAD-MS system to evaluate the best procedure.

Before the elution, the washing step was carried out in order to remove the interfering compounds non-specifically retained. The presence of non-specific binding sites is attributed to the high amount of monomer used during MIP synthesis by the non-covalent approach, and it was well known that polar and protic solvents weakened the non-covalent specific interactions between template and MIP. In this step, different washing solvents and volumes were investigated in this study: water, acetonitrile, methanol, and mixtures of water: $\mathrm{ACN}$ (80:20 and 90:10). The results indicated that best MIP recoveries and purification effect were achieved when water was used as the washing solvent (see Figure 5). Washing volume was also optimized and a volume of $1 \mathrm{~mL}$ of water was used as the optimum washing condition. On the other hand, the different recoveries obtained for NIP and MIP indicated that the interactions between the template and MIP were specific. In the wash step, analyte is lost when NIP is used as an adsorbent.

To ensure complete elution of the adsorbed analyte from the MISPE procedure, it is necessary to find out the appropriate solvent. Consequently, the efficacy of various solvents for the elution of 4-HPA was evaluated. The solvents tested were $\mathrm{MeOH}$ and ACN, with or without $1 \%$ formic, mixtures of both in different proportions (20:80; 50:50; $80: 20)$. $\mathrm{MeOH}$ with acetic acid in proportion (8:2) was also tested. The best recovery was obtained using acetonitrile with $1 \%$ formic acid. Different volumes of elution have also been tested. These studies revealed that the optimum volume of elution was $1.5 \mathrm{~mL}$. In urine samples, the recovery obtained from 4-HPA under the MISPE optimized conditions were of $94 \%$ (RSD 1.2). 


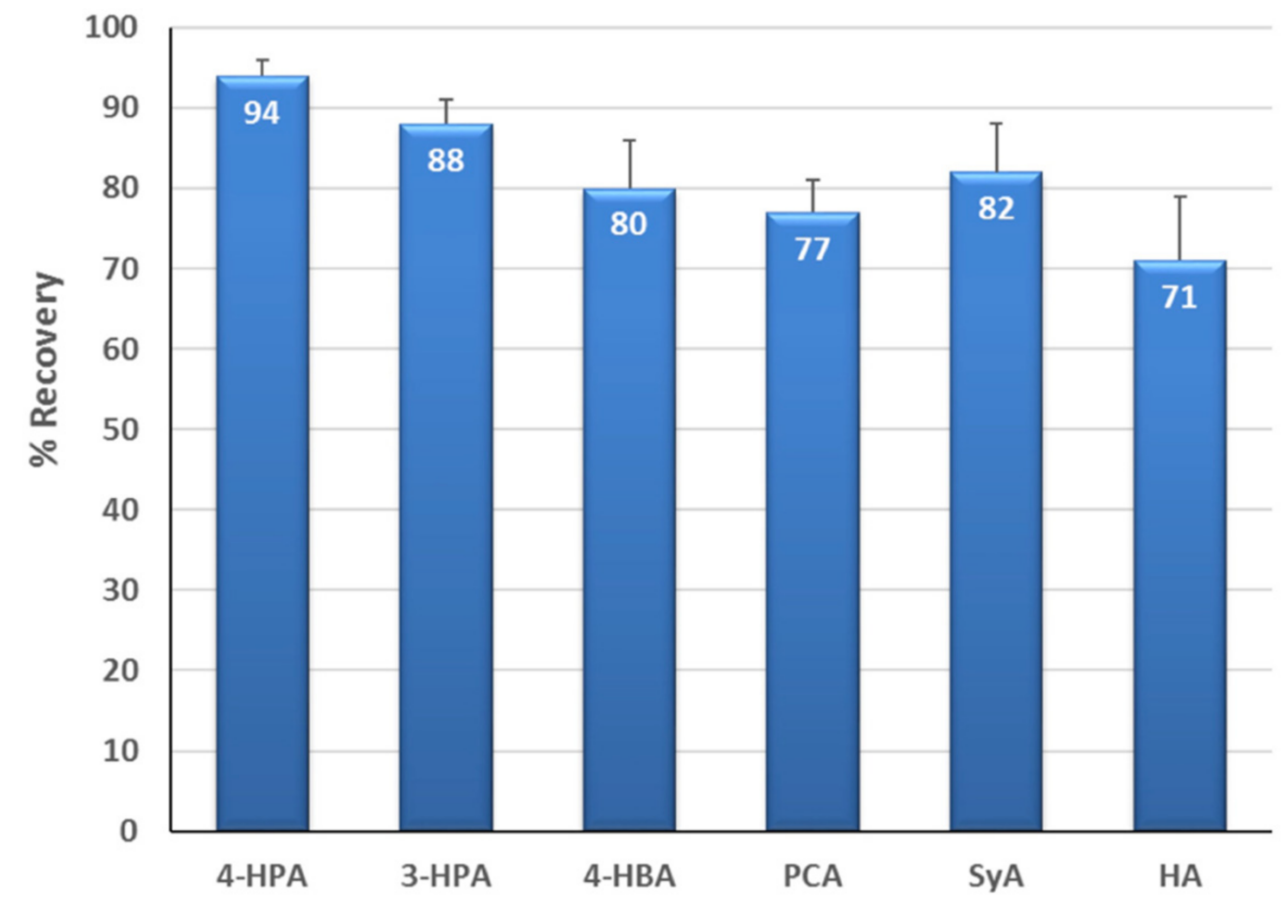

Figure 5. Recoveries percentage of the phenolic acid analogues obtained from the MISPE procedure. In the urine sample, the concentration of each phenolic acid was $4 \mathrm{mg} / \mathrm{L}$. Error bars indicate the standard deviation of replicates $(n=3)$.

\subsection{Validation of the MISPE Procedure}

The MISPE-LC-DAD-MS method developed was validated in terms of linearity, reproducibility, and detection and quantification limits (LOD and LQO), and average recoveries for 4-HPA under the optimized experimental conditions. Those were determined using spiked blank urine samples, prepared as previously has been described. Calibration curves were obtained using ten different concentrations (range from $0.25-40 \mathrm{mg} / \mathrm{L}$ ). The reproducibility of method, which was evaluated with the relative standard deviation parameter (RSD) in term of reproducibility (inter-day precision and intra-day precision) at three levels of fortification: $2 \mathrm{mg} / \mathrm{L}, 20 \mathrm{mg} / \mathrm{L}$, and $40 \mathrm{mg} / \mathrm{L}$. The intra-day precision was demonstrated at these three levels and RSD as well. Results are summarized in Table 2. Finally, the recovery values were more than $94 \%$ (RSD $1.2 \%$ ) over the range of concentrations tested.

Table 2. Validation parameter of the MISPE procedure.

\begin{tabular}{|c|c|c|c|c|c|c|c|c|c|}
\hline \multirow{3}{*}{ Method } & \multirow{3}{*}{$\mathbf{r}^{2}$} & \multirow{3}{*}{$\begin{array}{l}\text { LOD } \\
\mathrm{mg} / \mathrm{L}\end{array}$} & \multirow{3}{*}{$\begin{array}{l}\mathrm{LOQ} \\
\mathrm{mg} / \mathrm{L}\end{array}$} & \multicolumn{6}{|c|}{ Reproducibility (RSD) } \\
\hline & & & & \multicolumn{3}{|c|}{ Inter-Day Precision } & \multicolumn{3}{|c|}{ Intra-Day Precision } \\
\hline & & & & $2 \mathrm{mg} / \mathrm{L}$ & $20 \mathrm{mg} / \mathrm{L}$ & $40 \mathrm{mg} / \mathrm{L}$ & $2 \mathrm{mg} / \mathrm{L}$ & $20 \mathrm{mg} / \mathrm{L}$ & $40 \mathrm{mg} / \mathrm{L}$ \\
\hline MISPE-LC-DAD-MS & 0.990 & 1.22 & 3.69 & $1.6 \%$ & $2.8 \%$ & $2.8 \%$ & $6.7 \%$ & $3.2 \%$ & $3.1 \%$ \\
\hline
\end{tabular}

\subsection{Selectivity of MIP}

The complete confirmation of the imprinting performance involves the study of the selectivity of the MIP. This was accomplished by determination of binding capacity of the MIP in presence of other structural analogues of the 4-HPA.

The MISPE procedure was assessed mixing all studies analytes. In the urine sample, the concentration of each phenolic acid was $4 \mathrm{mg} / \mathrm{L}$. Figure 5 shows the comparative recoveries obtained for each. Experiments studying the retention on the NIP in the same conditions than the MIP were done, but the result on NIP were inconsiderable in comparison to MIP. 
MIP shows a highly specific molecular recognition for the template $(94 \%$ of recovery, RSD 1.2\%) over similar analogues. Molecular recognition of the compounds was influenced by the presence, number, and position of hydroxyl and carboxyl groups in the aromatic ring $[16,17,29,31]$. From the compounds tested 3-HPA, isomer of 4-HPA, shows the best structural resemblance, as its only difference is the hydroxyl group position, and recovery value was $88 \%$. All the compounds bear carboxyl and multiple hydroxyl groups being for this reason prone to forming hydrogen bonds with the polymer matrix. Accordingly, strong competition for specific and non-specific binding sites is expected. Moreover, this did not significantly affect the recoveries values of 4-HPA. The retention order of the compounds is associated with their potency to form strong hydrogen bonds and therefore follows the order of the number of hydroxyl and carboxyl groups. Syringic acid (82\%) and 4-HBA $(80 \%)$ are strongly retained, followed by PCA $(77 \%)$, and HA as the less retained $(71 \%)$. In addition, the molecular size also conditioned the recoveries values $[16,17,29,31]$.

These results show that 4-HPA-MIP is an effective sorbent and was successfully applied to the extraction of 4-HPA and other phenolic acids. On the other hand, the advantages of MIP as the selective sorbent was demonstrated because it provides greater specificity compared to other sorbents.

The selective extraction of these metabolites helps improve the application and results obtained by other less sensitive analysis methods than the validation method. It also facilitates the development of new screening methods as electrochemical sensors to estimate polyphenol excretion (unpublished authors work).

\section{Conclusions}

In this study, a molecularly imprinted polymer against 4-HPA was synthesized and applied as the selective sorbent in SPE for the treatment of human urine samples prior analysis. The results indicated that the MISPE proposed method had high recoveries, low detection limit, and good repeatability, providing a specific, reliable, and alternative method to determine phenolic acid, secondary metabolites of dietary flavonoid compounds, in human urine samples. In light of the above, we have developed a rapid and straightforward sample preparation. The proposed MISPE procedure can be seen as a specific and simple tool for the studies of bioavailability of dietary flavonoid compounds. MIP applications in the analytics determination of phenolic acid have been limited. In our knowledge, it is the first time that a MISPE procedure is applied to analyze urinary catabolites of a polyphenol compound.

Author Contributions: Research design, D.G.-G., J.S.J. and A.G.-P.; measurements and experiments, M.M.-G.; development, analysis of data, and presentation for results, M.M.-G. and A.G.-P.; writingoriginal draft preparation, M.M.-G.; writing-review and editing, D.G.-G., J.S.J. and A.G.-P. All authors have read and agreed to the published version of the manuscript.

Funding: This research received no external funding.

Institutional Review Board Statement: Not applicable.

Informed Consent Statement: Not applicable.

Data Availability Statement: The data presented in this study are available on request from the corresponding author.

Acknowledgments: Authors thank Ángel Maroto from the Department of Inorganic Chemistry and Technical Chemistry, and Department of Mathematical Physics and Fluids, Faculty of Sciences, National University of Distance Education (UNED), for the SEM and BET studies.

Conflicts of Interest: The authors declare no conflict of interest. 


\section{References}

1. Williamson, G.; Kay, C.D.; Crozier, A. The bioavailability, transport, and bioactivity of dietary flavonoids: A review from a historical perspective. Compr. Rev. Food Sci. Food Saf. 2018, 17, 1054-1112. [CrossRef] [PubMed]

2. Achaintre, D.; Buleté, A.; Cren-Olivé, C.; Li, L.; Rinaldi, S.; Scalbert, A. Differential isotope labeling of 38 dietary polyphenols and their quantification in urine by liquid chromatography electrospray ionization tandem mass spectrometry. Anal. Chem. 2016, 88, 2637-2644. [CrossRef]

3. Penczynski, K.J.; Krupp, D.; Bring, A.; Bolzenius, K.; Remer, T.; Buyken, A.E. Relative validation of 24-h urinary hippuric acid excretion as a biomarker for dietary flavonoid intake from fruit and vegetables in healthy adolescents. Eur. J. Nutr. 2017, 56, 757-766. [CrossRef]

4. Pérez-Jiménez, J.; Hubert, J.; Hooper, L.; Cassidy, A.; Manach, C.; Williamson, G.; Scalbert, A. Urinary metabolites as biomarkers of polyphenol intake in humans: A systematic review. Am. J. Clin. Nutr. 2010, 92, 801-809. [CrossRef] [PubMed]

5. Ulaszewska, M.M.; Trost, K.; Stanstrup, J.; Tuohy, K.M.; Franceschi, P.; Chong, M.F.F.; George, T.; Minihane, A.M.; Lovegrove, J.A.; Mattivi, F. Urinary metabolomic profiling to identify biomarkers of a flavonoid-rich and flavonoid-poor fruits and vegetables diet in adults: The FLAVURS trial. Metabolomics 2016, 12. [CrossRef]

6. De Rijke, E.; Out, P.; Niessen, W.M.A.; Ariese, F.; Gooijer, C.; Brinkman, U.A.T. Analytical separation and detection methods for flavonoids. J. Chromatogr. A 2006, 1112, 31-63. [CrossRef] [PubMed]

7. Pereira-Caro, G.; Ludwig, I.A.; Polyviou, T.; Malkova, D.; Garcia, A.; Moreno-Rojas, J.M.; Crozier, A. Identification of plasma and urinary metabolites and catabolites derived from orange juice (poly)phenols: Analysis by high-performance liquid chromatography-high-resolution mass spectrometry. J. Agric. Food Chem. 2016, 64, 5724-5735. [CrossRef]

8. Mora-Granados, M.; González-Gómez, D.; Gallego-Picó, A. Feasibility of the determination of three flavan-3-ols metabolites in urine samples via parallel factor analysis of fluorescence emission matrices. J. Funct. Foods 2017, 37, 303-309. [CrossRef]

9. Casado, N.; Morante-Zarcero, S.; Pérez-Quintanilla, D.; Cámara, J.S.; Sierra, I. Two novel strategies in food sample preparation for the analysis of dietary polyphenols: Micro-extraction techniques and new silica-based sorbent materials. Trends Food Sci. Tech. 2020, 98, 167-180. [CrossRef]

10. Dmitrienko, S.G.; Irkha, V.V.; Duisebaeva, T.B.; Mikhailik, Y.V.; Zolotov, Y.A. Synthesis and study of the sorption properties of 4-hydroxybenzoic acid-imprinted polymers. J. Anal. Chem. 2006, 61, 14-19. [CrossRef]

11. Dmitrienko, S.G.; Kudrinskaya, V.A.; Apyari, V.V. Methods of extraction, preconcentration, and determination of quercetin. J. Anal. Chem. 2012, 67, 299-311. [CrossRef]

12. Karasova, G.; Lehotay, J.; Klodzinska, E.; Gadzala-Kopciuch, R.; Buszewski, B. Comparison of several extraction methods for the isolation of benzoic acid derivatives from Melissa officinalis. J. Liq. Chrom. Relat. Tech. 2006, 29, 1633-1644. [CrossRef]

13. Karasova, G.; Lehotay, J.; Sadecka, J.; Skacani, I.; Lachova, M. Selective extraction of derivates of p-hydroxy-benzoic acid from plant material by using a molecularly imprinted polymer. J. Sep. Sci. 2005, 28, 2468-2476. [CrossRef]

14. Chen, F.F.; Wang, G.Y.; Shi, Y.P. Molecularly imprinted polymer microspheres for solid-phase extraction of protocatechuic acid in Rhizoma homalomenae. J. Sep. Sci. 2011, 34, 2602-2610. [CrossRef]

15. Denderz, N.; Lehotay, J. Using of molecularly imprinted polymers for determination of gallic and protocatechuic acids in red wines by high performance liquid chromatography. J. Chromatogr. A 2014, 1372, 72-80. [CrossRef]

16. Denderz, N.; Lehotay, J. Application of the van't Hoff dependences in the characterization of molecularly imprinted polymers for some phenolic acids. J. Chromatogr. A 2012, 1268, 44-52. [CrossRef] [PubMed]

17. Dmitrienko, S.G.; Irkha, V.V.; Apyari, V.V.; Klokova, E.V.; Zolotov, Y.A. Recognition of hydroxybenzoic acids and their esters by molecularly imprinted polymers. Mendeleev Commun. 2008, 18, 315-317. [CrossRef]

18. Michailof, C.; Manesiotis, P.; Panayiotou, C. Synthesis of caffeic acid and p-hydroxybenzoic acid molecularly imprinted polymers and their application for the selective extraction of polyphenols from olive mill waste waters. J. Chromatogr. A 2008, 1182, 25-33. [CrossRef] [PubMed]

19. Sun, B.W.; Li, Y.Z.; Chang, W.B. Molecularly imprinted polymer using p-hydroxybenzoic acid, p-hydroxyphenylacetic acid and p-hydroxyphenylpropionic acid as templates. J. Mol. Recognit. 2001, 14, 388-392. [CrossRef] [PubMed]

20. Li, N.; Ng, T.B.; Wong, J.H.; Qiao, J.X.; Zhang, Y.N.; Zhou, R.; Chen, R.R.; Liu, F. Separation and purification of the antioxidant compounds, caffeic acid phenethyl ester and caffeic acid from mushrooms by molecularly imprinted polymer. Food Chem. 2013, 139, 1161-1167. [CrossRef] [PubMed]

21. Sun, B.W.; Wu, L.Q.; Li, Y.Z. Experimental and theoretical study on p-hydroxybenzoic acid imprinted polymers with different functional monomers. Acta Chim. Sin. 2004, 62, 598-602.

22. Boscari, C.N.; Mazzuia, G.R.; Wisniewski, C.; Borges, K.B.; Figueiredo, E.C. Molecularly imprinted probe for solid-phase extraction of hippuric and 4-methylhippuric acids directly from human urine samples followed by MEKC analysis. Electrophoresis 2017, 38, 1083-1090. [CrossRef] [PubMed]

23. Ye, L.; Mosbach, K. Molecularly imprinted microspheres as antibody binding mimics. React. Funct. Polym. 2001, 48, 149-157. [CrossRef]

24. Cieplak, M.; Kutner, W. Artificial Biosensors: How Can Molecular Imprinting Mimic Biorecognition? Trends Biotechnol. 2016, 34, 922-941. [CrossRef]

25. De Ferrars, R.M.; Czank, C.; Zhang, Q.; Botting, N.P.; Kroon, P.A.; Cassidy, A.; Kay, C.D. The pharmacokinetics of anthocyanins and their metabolites in humans. Br. J. Pharmacol. 2014, 171, 3268-3282. [CrossRef] [PubMed] 
26. Nash, V.; Ranadheera, C.S.; Georgousopoulou, E.N.; Mellor, D.D.; Panagiotakos, D.B.; McKune, A.J.; Kellett, J.; Naumovski, N. The effects of grape and red wine polyphenols on gut microbiota-A systematic review. Food Res. Int. 2018, 113, 277-287. [CrossRef] [PubMed]

27. Sasot, G.; Martínez-Huelamo, M.; Vallverdu-Queralt, A.; Mercader-Martí, M.; Estruch, R.; Lamuela-Raventós, R.M. Identification of phenolic metabolites in human urine after the intake of a functional food made from grape extract by a high resolution LTQ-Orbitrap-MS approach. Food Res. Int. 2017, 100, 435-444. [CrossRef]

28. De Middeleer, G.; Dubruel, P.; De Saeger, S. Characterization of MIP and MIP functionalized surfaces: Current state-of-the-art. Trac-Trends Anal. Chem. 2016, 76, 71-85. [CrossRef]

29. Shi, S.Y.; Guo, J.F.; You, Q.P.; Chen, X.Q.; Zhang, Y.P. Selective and simultaneous extraction and determination of hydroxybenzoic acids in aqueous solution by magnetic molecularly imprinted polymers. Chem. Eng. J. 2014, 243, 485-493. [CrossRef]

30. Baggiani, C.; Giraudi, G.; Giovannoli, C.; Tozzi, C.; Anfossi, L. Adsorption isotherms of a molecular imprinted polymer prepared in the presence of a polymerisable template-Indirect evidence of the formation of template clusters in the binding site. Anal. Chim. Acta 2004, 504, 43-52. [CrossRef]

31. Schwarz, L.J.; Danylec, B.; Harris, S.J.; Boysen, R.I.; Hearn, M.T.W. Preparation of molecularly imprinted polymers for the selective recognition of the bioactive polyphenol, (E)-resveratrol. J. Chromatogr. A 2011, 1218, 2189-2195. [CrossRef] [PubMed] 